\title{
Influencing Factors of Transverse Interface Cracking of Carbon Fiber Reinforced Composite
}

\author{
Liang $\mathrm{Wu}^{1, \mathrm{a}}$, Chenghong Duan ${ }^{1, \mathrm{~b}}$, Dan $\mathrm{Hu}^{1, \mathrm{c}}$ \\ ${ }^{1} \mathrm{CAE}$, Beijing University of Chemical Technology, Beijing, 100029, China \\ anickwuliang@gmail.com, bduanchenghong@163.com, chudannn@163.com
}

Key Words: interface strength, interface stiffness, volume fraction, XFEM

\begin{abstract}
Usually under a transverse load large enough, interface cracking and matrix fracture will occur to the unidirectional carbon fiber composite. Based on the extended finite element method (XFEM) an d the Surface-based cohesive behavior provided by ABAQUS, some factors, such as the volume fraction of carbon fiber, interface strength and stiffness, were considered to study their effects on the transverse performance of the carbon fiber composite. Which provides a reference for the study of fiber matrix interface and transverse mechanical properties.
\end{abstract}

\section{Introduction}

The transverse mechanical properties of unidirectional carbon fiber composites are much lower than those of longitudinal mechanical properties due to fiber orientation and fiber matrix interface. It is the key to improve the transverse mechanical properties of carbon fiber composites by studying the fracture process of carbon fiber composites under transverse load and analyzing the influence of interface and other factors on the transverse mechanical properties of carbon fiber composites.

In the aspect of the transverse fracture and the performance of the composites, the domestic and foreign scholars have made a lot of achievements. Shuai $\mathrm{Wu}$ et al [1] explored the surface structure's effects on the tensile strength, strain and interface formation. Carbon fiber without obvious microvoids or surface defects tended to have better tensile strain but form weak interface with pyrocarbon, leading to a better pseudo-ductility and ability to absorb more fracture energy under load. Christopher Baker et al [2] analyzed transverse cracks in CFRP composites by modal acoustic emission and peak frequencies. Studies have shown that the location of the $90{ }^{\circ}$ layer directly affects lateral crack formation and stress distribution. Xiaoqiang Wang et al [5] studied the interface property's effects on the composite transverse effective property and damage process with the representative volume element model. Marcin Kaminski [4] conducted a multi-scale finite element simulation of the structural inhomogeneity and interfacial defects of unidirectional periodic composites by wavelet analysis. Nan Zheng et al [5] used the silane coupling agent (SCA) to improve the carbon fiber and epoxy resin interface oxidation performance, thereby improving the material properties. Chen Wang et al [6] showed that the tensile strength of the fibers was improved not only by the surface treatment, but also by the improvement of the fracture toughness at the fiber-matrix interface.

In this paper, ABAQUS software was used to simulate the transverse fracture process of carbon fiber composites under different conditions, and the effects of common factors on the transverse tensile process of the composites were investigated.

\section{Effects of several factors on loading process}

The effects of the carbon fiber volume fraction, interface strength and stiffness on the composite transverse loading process are studied in detail.

In order to improve the computational efficiency and converge readily, the $1 / 2$ model as shown in Fig. 1 is taken to perform the following analysis. Symmetrical constraint is applied to the symmetry edge a, for edge b and c their normal displacements are constraint, and the upward displacement load is applied to edge $\mathrm{d}$. 

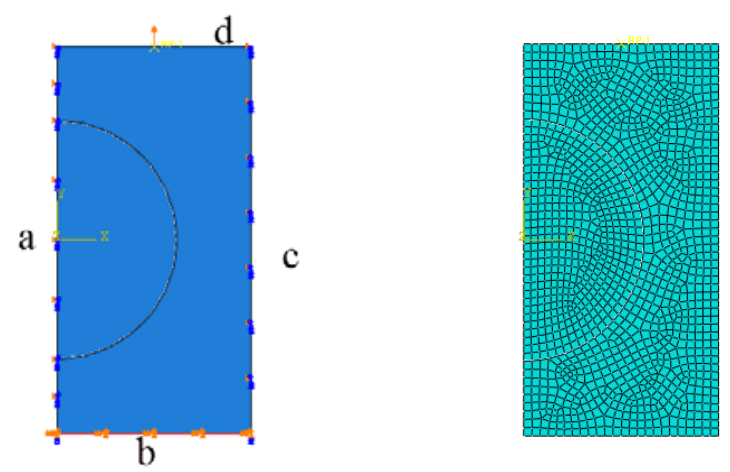

Fig. 1 1/2 model

\subsection{Effect of carbon fiber volume fraction}

The carbon fiber volume fraction is an essential factor to the composite mechanical property. Usually more fiber means higher longitudinal strength and modulus, but it introduces more interfaces at the same time. The increasing of the interface fraction will inevitably bring more occurrence possibility of crack and initial defects. Now five different cases with diverse fiber fraction (30\%, 40\%, 50\%, 60\%, 70\%) are analyzed.

The simulation result demonstrate that the five cases' fracture modes are basically the same. Fig. 2 shows the load-displacement curves of the five different carbon fiber fraction model.

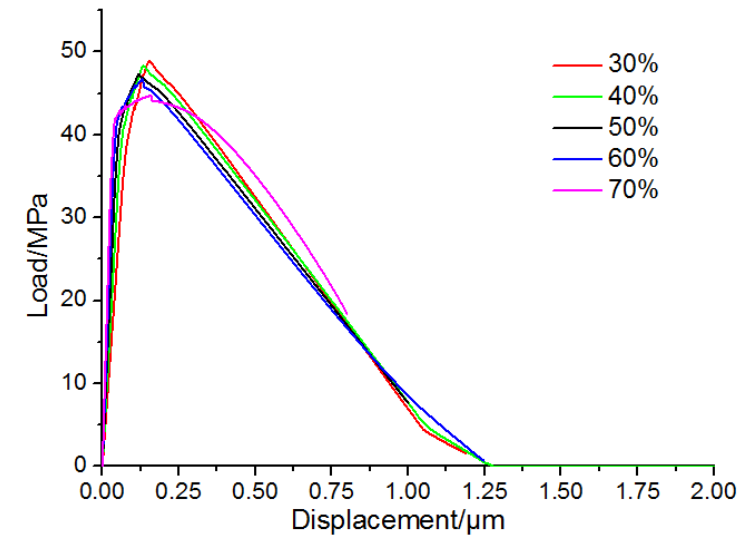

Fig.2 Load-displacement curves of models with different fiber fraction

It can be seen that, in the elastic stage the curve slope increases as the fiber fraction increases, i.e. higher fiber fraction means higher transverse modulus. However the transverse strength (i.e. the maximum load value in each curve) become lower while the fiber fraction increases, see Fig.3. That means under the transverse load, the strengthening effect of the fiber is quite limited, when the fiber fraction increases the matrix fraction decreases, as a result the scattered fine cracks will connect to each other more easily, and that leads to the final macroscopical facture of the material.

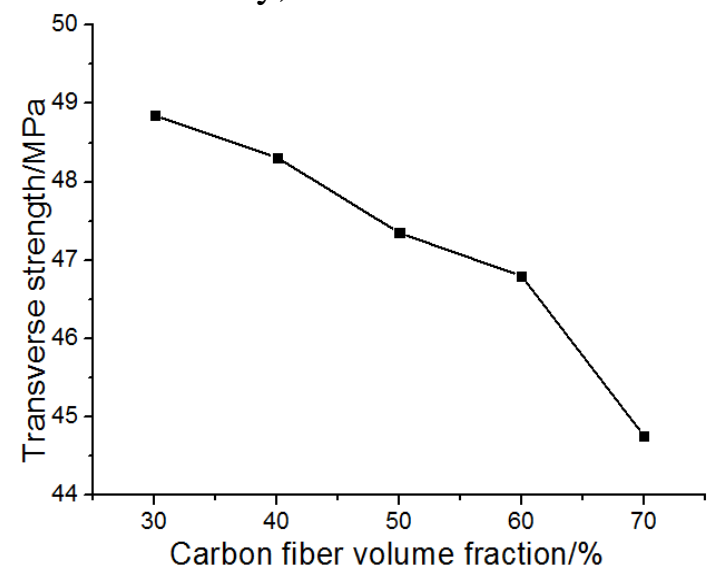

Fig.3 Transverse strength-fiber fraction curve

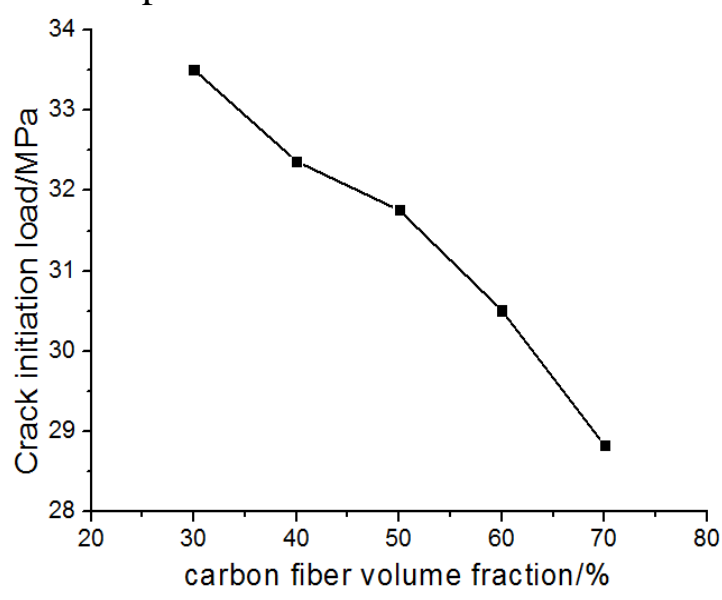

Fig.4 Crack initiation load-fiber fraction curve 
Fig.4 shows the crack initiation load's variation trend as the fiber fraction increasing. Crack initiation load is the load value at the moment that the crack just begins, it reflects that how difficult the cracking occurs. It can be seen that the crack initiation load nearly declines linearly as the fiber fraction increases.

\subsection{Effect of interface strength}

Take the model with $50 \%$ carbon fiber fraction as an example, to analyze the effect of the interface strength.

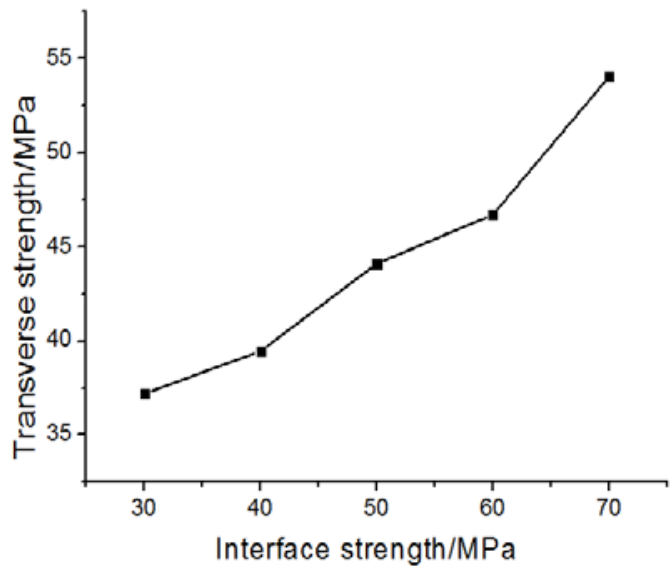

Fig. 5 Transverse strength-interface strength curve

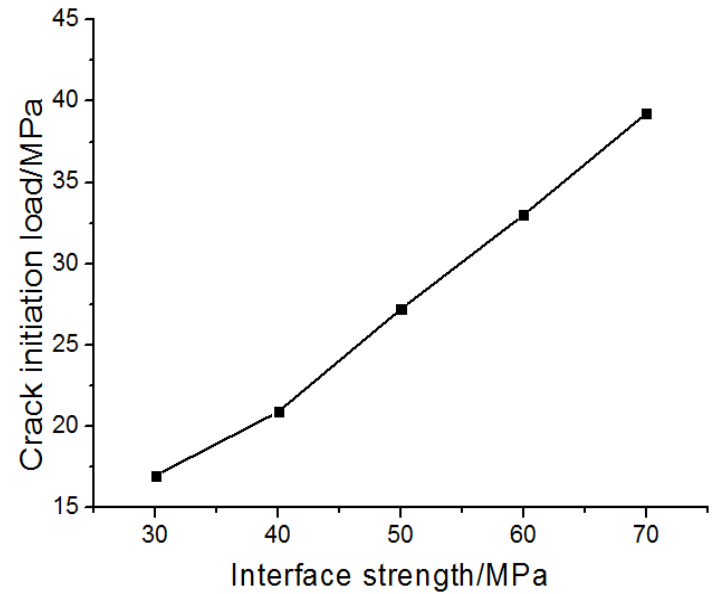

Fig.6 Crack initiation load-interface strength curve

Fig.5 and Fig.6 respectively shows the variation trends of transverse strength and crack initiation load as the fiber fraction increasing. Both the transverse strength and crack initiation load increase observably as the interface strength increases. That means improving the interface cohesive strength is a most effective way to avoid cracking and improve the material transverse strength.

\subsection{Effect of interface stiffness}

The interface is defined as Eq.1, where $\mathrm{E}$ is the elastic modulus, $\mathrm{t}$ is the interface thickness. Stiffness reflects the deformation resistance.

$$
K=\frac{E}{t}
$$

Assume the normal and shear stiffness of the interface is the same, also take the model with $50 \%$ carbon fiber fraction as an example, to analyze the effect of the interface stiffness.

According to the simulation result, the matrix crack location with higher interface stiffness is on the upper side of the model, while in the model with lower interface stiffness the matrix crack appears in the middle of the model. As shown in Fig.7, their crack paths are different.

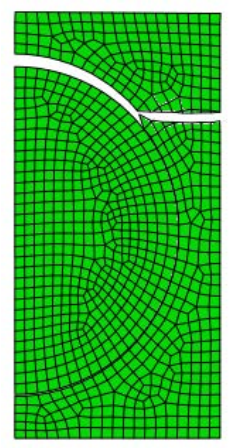

(a) $8000 \mathrm{GPa} / \mathrm{mm}$

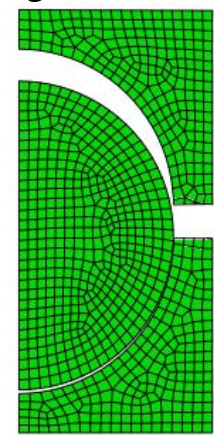

(b) $250 \mathrm{GPa} / \mathrm{mm}$

Fig.7 Cracks of models with interface stiffness $8000 \mathrm{GPa} / \mathrm{mm}$ and 250GPa/mm

When the interface stiffness is low, the interface can produce large deformation on the load direction without damage, so the load cannot be transferred to the fiber effectively, and the matrix 
bears most of the load. That leads to the matrix fracture at the most narrow cross section of it. When the interface stiffness is high, load can be transferred to the fiber quickly, and the fiber bear the load mostly. Till the stress at the interface exceed the interface strength then interface local cracking happens, so that the stress in the matrix near the interface crack tip become high enough to crack subsequently.

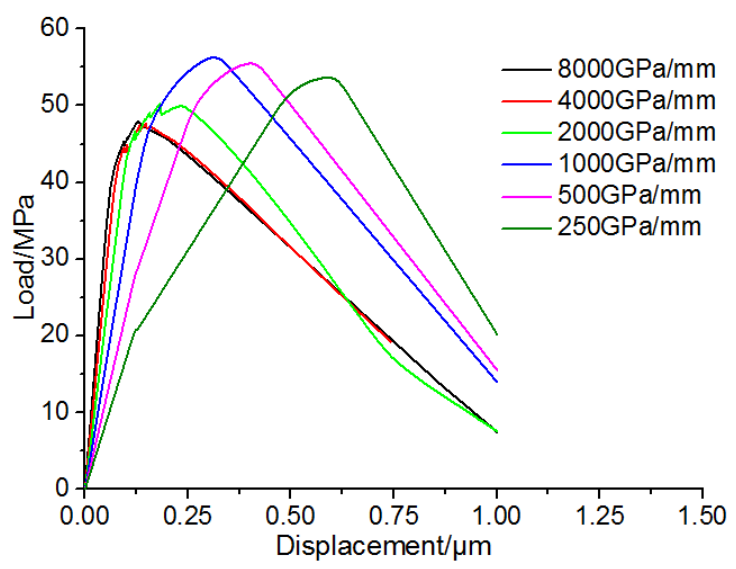

Fig.8 Load-displacement curves of models with different interface stiffness

From Fig.8 it can be seen that the interface stiffness has influences on every stage of the loading process. As to the transverse strength of the model, if the interface stiffness is too low, crack begins in the matrix firstly, so the transverse strength is low; if the interface stiffness is too high, the composite tends to be brittle, the transverse strength still low. As shown in Fig.9, the transverse strength of the model increases first and then declines as the interface stiffness increases, especially when the stiffness is $1000 \mathrm{GPa} / \mathrm{mm}$ the transverse strength reaches the maximum value. In Fig.10, the initiation crack load increases first as the interface stiffness increases, and it stays around $40 \mathrm{MPa}$ after the stiffness exceeds $1000 \mathrm{GPa} / \mathrm{mm}$.

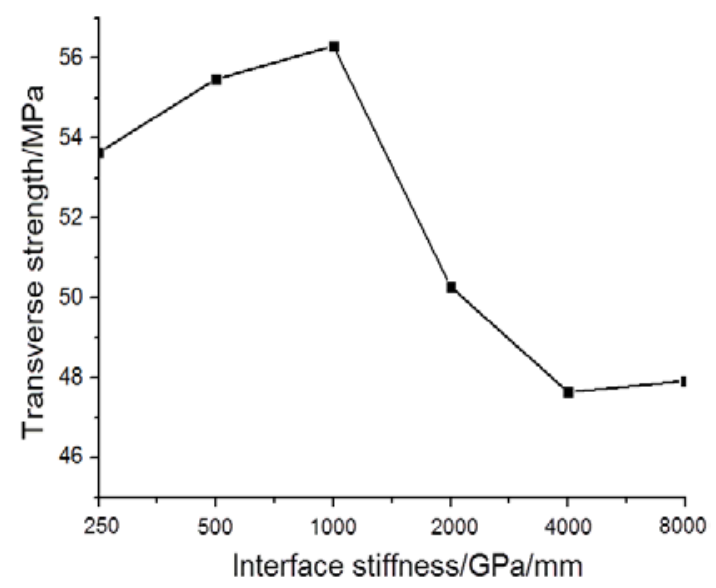

Fig.9 Transverse strengths of models with different interface stiffness

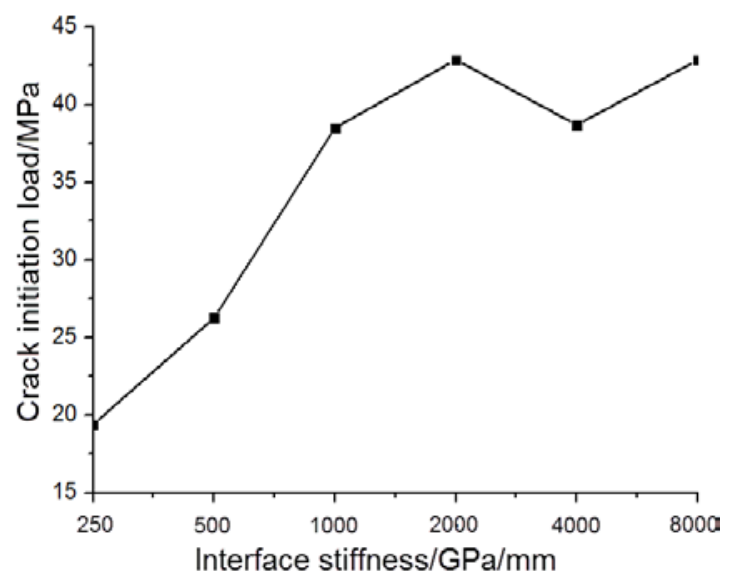

Fig.10 Crack initiation loads of models with different interface stiffness

\section{Conclusion}

Based on the XFEM and the Surface-based cohesive behavior provided by ABAQUS, the effect of several factors on the transverse performance was investigated by simulating the interface and matrix transverse cracking process in carbon fiber reinforced composite.

Increasing the carbon fiber volume fraction makes the composite transverse strength decline and the crack begin more easily. Improving the interface strength is a most effective way to prevent cracking and enhance the composite transvers strength. Interface stiffness is very essential to the deformation compatibility and load transferring between the fiber and matrix. If the interface stiffness is too low, cracks begins firstly in the matrix, the transverse strength and initiation crack of 
the composite will be low. If the stiffness of the interface is too high, it will make the compatible deformation capacity decline, so the composite tends to be brittle. The crack initiation load turns to be stable as the interface stiffness become high enough.

\section{References}

[1] Wu S, Liu Y, Ge Y, et al. Surface structures of PAN-based carbon fibers and their influences on the interface formation and mechanical properties of carbon-carbon composites[J]. Composites Part A: Applied Science and Manufacturing, 2016, 90: 480-488.

[2] Baker C, Morscher G N, Pujar V V, et al. Transverse cracking in carbon fiber reinforced polymer composites: Modal acoustic emission and peak frequency analysis[J]. Composites Science and Technology, 2015, 116: 26-32.

[3] Xiaoqiang Wang, Jifeng Zhang, Zhenqing Wang, Song Zhou, Xinyang Sun. Effects of interphase properties in unidirectional fiber reinforced composite materials [J]. Materials and Design, 2011, 32: 3486-3492.

[4] Marcin Kaminski. Interface defects in unidirectional composites by multiresolutional finite element analysis[J]. Computers and Structures, 2006, 84: 1190-1199

[5] Zheng N, He J, Zhao D, et al. Improvement of atomic oxygen erosion resistance of carbon fiber and carbon fiber/epoxy composite interface with a silane coupling agent[J]. Materials \& Design, 2016, 109: 171-178.

[6] Wang C, Ji X, Roy A, et al. Shear strength and fracture toughness of carbon fibre/epoxy interface: effect of surface treatment[J]. Materials \& Design, 2015, 85: 800-807. 